\title{
Motor neuron disease: the impact of decreased speech intelligibility on marital communication
}

\section{Abstract}

Background: The onset of motor neuron disease (MND), a neurodegenerative disease, results in physical and communication disabilities that impinge on an individual's ability to remain functionally independent. Multiple aspects of the marital relationship are affected by the continuously changing roles and responsibilities. Communication is one of the most constructive ways of dealing with emotions that are elicited by these changes.

Method: This study explored the association between the deteriorating speech of persons with MND and couples' perception of marital communication. Fourteen couples participated in this non-experimental correlational research study. Data were collected over a 12-month period through the administration of objective and subjective measures.

Results: Results showed that despite decreased speech intelligibility, the relationship between the deteriorating speech and the couples' perception of marital communication was not statistically significant.

Conclusion: Overall, the results proved that the supposition that communication between couples will invariably deteriorate as a result of progressively reduced speech intelligibility is not necessarily valid. The fundamental importance of effective communication in marriage is highlighted. It is well established that augmentative and alternative communication strategies can preserve the ability to develop and maintain intimate rewarding relationships, even in the face of profound physical disabilities.

\section{Introduction}

The lives of adults with acquired disorders and those of their spouses are irreversibly altered as a consequence of the onset of a progressive, degenerative illness, such as motor neuron disease (MND). MND usually results in severe disability involving all four limbs, speech, swallowing and eventually breathing. ${ }^{1}$ MND has an incidence of 1.5-2.5 per 100000 of the population worldwide and predominantly affects middle-aged and elderly people, with a mean age of 65 years. ${ }^{2}$ Average MND survival is generally reported to range between two to five years. ${ }^{3}$

The International Classification of Functioning, Disability and Health (ICF) provides a framework to describe the impact of MND on levels of activity, participation, and environmental and personal factors. ${ }^{4}$ Persons with MND will invariably experience a progressive decline in physical and communication abilities. Relentless progressive muscular weakness will negatively impact on individuals' ability to perform many physical activities of daily living until ultimately, dependence is inevitable. ${ }^{5}$ However, one of the most profound changes that persons with MND will experience is loss of their speech. ${ }^{6}$ The rapid decline of speech function as a result of dysarthria is common as $75 \%$ per cent of persons with MND will reach a point where intelligible verbal communication is no longer possible. ${ }^{7}$ Dysarthria, a motor speech disorder that results from damage to the peripheral or central nervous system is characterised by the imprecision of the movement of speech musculature, resulting in decreased speech intelligibility. ${ }^{8}$ Typically, speaking rate is initially slower while speech intelligibility remains relatively high, but as the disease progresses and dysarthria becomes more apparent, speech intelligibility decreases dramatically. ${ }^{9}$

Speech intelligibility is seen as a significant factor in determining communication effectiveness ${ }^{10}$ and is a critical aspect in the assessment and monitoring of speech in persons with MND. ${ }^{5}$ Communication effectiveness implies that an individual has an adequate level of communication to meet important communication goals. ${ }^{11}$ 
The positive relationship between speech intelligibility and communication effectiveness in MND has been confirmed. ${ }^{7,12}$ This inevitable decline in the communication function of persons with MND implies that in $80 \%$ of cases, alternative communication strategies are required to support the daily communication needs of persons with MND and their families. ${ }^{7}$

Augmentative and alternative communication (AAC) strategies can preserve communication (and therefore participation in everyday life situations), even in the face of profound motor deterioration. ${ }^{13}$ AAC is acknowledged as best practice and is widely accepted among persons with MND and their families. ${ }^{14} \mathrm{~A}$ variety of strategies are successfully used by persons with MND to facilitate communication. These include unaided low-technology strategies (e.g. facial expression, eye gaze, gestures and sign language), aided low-technology strategies (e.g. topic and alphabet cues and alphabet boards), and high-technology strategies, such as dedicated AAC devices (e.g. speech-generating devices, absolute head-tracking and eye-tracking technology)..$^{14}$ In light of the significant changes in the motor abilities of people with MND, it is imperative that AAC strategies meet the changing physical needs as the disease progresses. ${ }^{9}$ Therefore, it is important that the recommended strategies support multiple, adjustable access methods that allow them to transition from hand access to scanning and eye pointing or tracking, for example. ${ }^{9}$

Given its prominence over physical abilities and function, communication is essential for the human experience and may be the single most important aspect for personal well-being. ${ }^{11}$ As chronic illness is a dyadic affair, marriage will predictably be affected by the diagnosis of MND. Multiple aspects of the couple's life will be affected and their relationship will never be the same again. They will experience similar psychosocial effects as they deal with their own distress and that of their partner. Facing the emotional trauma of losing their relationship elicits strong emotions in both members. Guilt, anger, resentment and frustration are often reported and couples facing the stress of chronic illness must find ways to manage these emotions. ${ }^{15}$ One of the most constructive ways of dealing with these emotions is communication. ${ }^{16}$

Sensitive, open, and direct communication plays a central role in marriage and is essential for couples to cope with chronic illness. ${ }^{15-17}$ The ability of spouses to listen, to express themselves clearly and accurately and to understand each other is critically important in marital communication. ${ }^{18}$ This is achieved by the key characteristics of marital communication, namely verbal (i.e. speech) and nonverbal communication (i.e. gestures, facial expressions and body language). Nonverbal cues qualify and colour verbal communication, but can also occur independently of it. Spouses, through their history of multiple shared interactions, are expected to become sensitive to each other's facial expressions, idiosyncratic gestures, vocalisations and feelings. ${ }^{18}$ Therefore, self-exploration, self-understanding and feelings of intense closeness are facilitated by communication.

Negative life events, such as the onset of illness and/or disability, disrupt the couples' communicative competence. With the onset of MND, couples' communicative competence is affected in a gradual way. ${ }^{17}$ Both members of the couple therefore need to move toward integrating AAC techniques into a new lifestyle and learn to become effective communicators in interactions that involve various AAC strategies. ${ }^{19}$ In the early stages of the communication disorder, people with MND rely on residual speech to facilitate social closeness with their spouse, but as their impairment become more severe, multiple communication strategies may be used for this purpose..$^{20}$ Interpreting the health and disability status of MND in the context of environmental and personal factors encourages a more holistic approach to intervention that is focused on maximizing residual function, altering the environment and educating families of people with MND to ensure that they are provided the opportunity to function optimally. ${ }^{4}$

As there is a dearth of available information on how the decline in speech intelligibility of persons with MND impacts on marital communication, the primary aim of this study was to compare how persons with MND and their spouses perceive changes in their marital communication in relation to the deteriorating speech of persons with MND. Firstly, the current study aimed to describe the communication abilities and speech intelligibility patterns of persons with MND, and secondly, to describe the perception of marital communication indicated by couples across the disease progression.

\section{Methodology}

\section{Research design}

A non-experimental correlational design was employed to study the relationship between the deteriorating speech of persons with MND and the couple's perception of marital communication at three visits at six-monthly intervals over a 12-month period.

\section{Participant description}

Fourteen couples $(n=28)$, divided into two groups, participated in the study. Participant group 1 included persons with MND $\left(n_{1}=14\right)$, and participant group 2, their spouses $\left(n_{2}=14\right)$. Participants were recruited using a non- 
probability, purposive sampling strategy.

\section{Participant group 1: Persons with motor neuron} disease

Only persons with MND with a neurologist-confirmed diagnosis of MND who were in established intimate relationships for at least 12 months prior to the onset of MND, and who had no reported speech or language, hearing or visual impairment other than those resulting from the MND, were included in the study. This group comprised 11 males and three females. Six participants presented with spinal-onset MND, six with bulbar-onset MND and two with mixed-onset MND. The average age of the participants was $57 ; 1$ (years, months) [range 44;1 to $70 ; 4$; standard deviation $(S D)=8 ; 67]$. The average time since the onset of the MND symptoms was $3 ; 76$ (range 3 months to $8 ; 6 ; \mathrm{SD}=2 ; 19$ ) and the average time since diagnosis was 2;37 (range 5 months to 7 years; $S D=2 ; 08$ ).

At the onset of the study, one participant's MND classification ${ }^{20}$ was mild; seven participants were classified as moderate, and six participants as severe. The disease progression was apparent at both the second and third visits (see Figure 1). The number of participants classified as "moderate" decreased to four at the second visit, while there was an increase in the number of participants classified as "severe" to nine, with one classified as "terminal". Attrition of participants, as a result of death shortly after the second visit decreased the sample size to only nine participants at

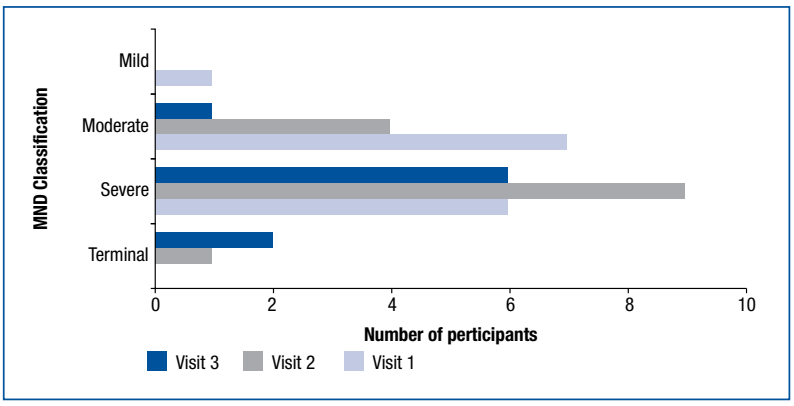

Figure 1: Motor neuron disease classification across visits

the third visit. Of these participants, only one was classified as "moderate", six as "severe" and two as "terminal".

\section{Participant group 2: Spouses}

The second participant group comprised the 14 spouses $\left(n_{2}\right.$ $=14$ ) of the persons with MND in participant group 1. There were 11 females and three males, and none reported any communication, vision and hearing difficulties that impacted on their daily living activities. The average age of the spouse group was $57 ; 8$ (range $47 ; 6$ to $70 ; 3, \mathrm{SD}=8 ; 64$ ). Thirteen of the couples were married for more than a decade, with an average of $29 ; 10$ (range 3 years to $45 ; 6 ; S D=13 ; 81$ ).

\section{Materials}

Five validated measures were utilised to meet the requirements posed by the research aims: classification of MND, ${ }^{21}$ Amyotrophic Lateral Sclerosis Severity Scale (ALSSS) Speech Scale, ${ }^{22}$ Sentence Intelligibility Test (SIT), ${ }^{23}$ Modified Communication Effectiveness Index (CETI-M) and the Primary Communication Inventory $(\mathrm{PCl}) .{ }^{24}$

\section{Classification of motor neuron disease ${ }^{20}$}

This classification system describes the severity of MND across the functional modalities of speech, mobility and the ability to use upper limbs for daily living activities. ${ }^{21}$ Classification ranges between four states: mild, moderate, severe and terminal.

\section{Amyotrophic Lateral Sclerosis Severity Scale ${ }^{22}$}

Information was obtained regarding the severity level in the four categories of swallowing, lower extremities, upper extremities, and speech ability.11,21 The ALSSS Speech Scale ${ }^{22}$ was used to describe the functional impairment experienced by persons with MND in the area of speech for this study. The 10-point scale of function includes five general categories namely normal speech processes, detectable speech disturbance, the need for behavioural modifications, the need for use of augmentative communication and loss of useful speech.

\section{Sentence Intelligibility Test $^{23}$}

This widely used standardised clinical transcription test was used to objectively measure speech intelligibility of the participants in group 1. A series of 11 randomly generated unrelated sentences, varying in length from five to 15 words, were read by participants at each visit. Transcription of the recorded responses yielded the percentage intelligible productions.

\section{Modified Communication Effectiveness Index ${ }^{5}$}

This measure of functional communication for persons with MND uses a visual analogue for 10 contextual situations on a 7-point Likert scale. The persons with MND and their spouses completed the CETI-M ${ }^{5}$ separately to determine perceived communication effectiveness in the same situations.

\section{Primary Communication Inventory ${ }^{24}$}

The $\mathrm{PCl},{ }^{24}$ a 25 -item instrument, was designed to assess marital communication. The $\mathrm{PCl}^{24}$ was completed separately by both members of the couple at all three visits. The overall score appears to be a reliable indicator of the soundness of communication between two members of a couple. ${ }^{24}$

\section{Procedures}

Ethics approval for the study was obtained from University of Pretoria's research ethics committee. Written informed 
consent was obtained from the MND Association and all participants, using established and approved methods. All participants in the study were fully informed of the nature of the study and were assured of confidentiality and their right to withdraw from the study at any time, without any negative consequences. Participants were visited at home at times indicated to be most convenient for both members of the couple. During the first visit, the aims of the research were explained and consent was obtained. Demographic information was acquired from the couple and the ALSSS Speech Scale ${ }^{22}$ and Classification of MND ${ }^{21}$ were completed by the researcher based on the clinical observations and interviews with both members of the couple. Persons with MND completed the following instruments with the assistance of the researcher: SIT, ${ }^{23}$ CETI-M ${ }^{5}$ and $\mathrm{PCl}^{24}$ The spouses completed the CETI- $\mathrm{M}^{5}$ and $\mathrm{PCl}^{24}$ privately after clear instructions were given. The two subsequent visits (visit 2 and visit 3) were scheduled at six-monthly intervals at which the same measuring instruments as described for visit 1 were administered.

\section{Data analysis}

Data were documented on all the relevant measuring instruments and encoded according to data definitions. Descriptive statistics were used to describe and analyse the data. The Friedman test was used with the repeated measures obtained from each participant group across the visits, while the Spearman rank correlation (Rho) was used to compute the correlation between speech intelligibility and communication effectiveness; as well as speech intelligibility and marital communication. The Wilcoxon test was used to compare the differences between the communication effectiveness ratings and marital communication scores of persons with MND and their spouses. In addition, Cohen's $d$ (effect size) was calculated to establish the size of the statistically significant difference.

\section{Results}

The first aim addressed the description of communication abilities and speech intelligibility patterns across the disease progression. The second aim addressed couples' perception of marital communication.

\section{Communication abilities}

Participants' functional impairment in the area of speech was established using the ALSSS Speech Scale.22 The progressive decline in speech function across all visits was evident (see Figure 2). At the last visit, only one of the nine participants reported a "detectable speech disturbance", three had to implement "behavioural modifications", three used "augmentative communication", and two reported "loss of useful speech". Participants who reported "use of

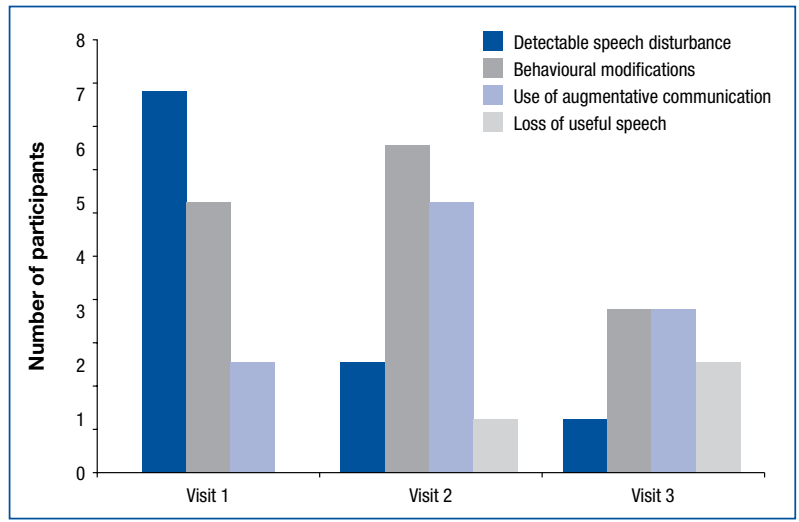

Figure 2: ALSSS scores across visits

augmentative communication" and "loss of useful speech" all facilitated communication with familiar and unfamiliar partners through facial expression and partner-assisted "yes" or "no" questions. During the last visit, none of the participants made use of high technology AAC devices for communication at the last visit due to a decline in their functional motor abilities.

\section{Speech intelligibility}

At the initial visit, the mean percentage speech intelligibility (as measured by the $\mathrm{SIT}^{23}$ ) for all participants was $75.58 \%$ (range 35 to 100; SD = 22.93), which decreased to $53.07 \%$ (range 0 to $93 ; \mathrm{SD}=32.81$ ) at the second visit. This continued to decrease to an average of $36.22 \%$ (range 0 to 73 ; SD = 24.6) at the last visit. A Friedman test (two-way analysis of variance) was employed to determine whether the change in speech intelligibility was statistically significant over time. A p-value of 0.0005 was noted, implying that there was a significant decrease in the speech intelligibility of the persons with MND across the disease progression (see Table I). Cohen's $d(d=0.9168)$ confirmed that the effect of the difference between the first and third visit was large. ${ }^{23}$

Table I: Percentage speech intelligibility across visits

\begin{tabular}{|l|c|c|c|}
\hline & $\begin{array}{c}\text { Visit 1 } \\
\mathbf{n = 1 4}\end{array}$ & $\begin{array}{c}\text { Visit 2 } \\
\mathbf{n = 1 4}\end{array}$ & $\begin{array}{c}\text { Visit 3 } \\
\mathbf{n = 9}\end{array}$ \\
\hline Mean & $75.58^{\mathrm{a}}$ & $53.07^{\mathrm{a}, \mathrm{b}}$ & $36.22^{\mathrm{b}}$ \\
\hline Standard deviation & 22.93 & 32.81 & 24.6 \\
\hline Median & 79.50 & 57.00 & 39.00 \\
\hline Cohen's $d$ & & 0.9168 (large) \\
\hline Friedman p-value & & $0.0005^{*}$ &
\end{tabular}

Means with different superscripts differ significantly at the $5 \%$ level ${ }^{*}$ Significance at the $5 \%$ level

\section{Communication effectiveness}

The CETI-M ${ }^{5}$ was completed by both the persons with MND (self-perceived) and spouses (listener-perceived) at each visit. Ten communication situations, ranging from speaking to familiar persons and strangers in a quiet and in a noisy environment, to talking on the telephone, were rated. $A$ 
Table II: Communication effectiveness $\left(\mathrm{CETI}-\mathrm{M}^{5}\right)$ ratings across visits and between participant groups

\begin{tabular}{|c|c|c|c|c|c|c|c|c|}
\hline & \multicolumn{2}{|c|}{$\begin{array}{l}\text { Visit } 1 \\
n=14 \\
\end{array}$} & \multicolumn{2}{|c|}{$\begin{array}{l}\text { Visit } 2 \\
n=14 \\
\end{array}$} & \multicolumn{2}{|c|}{$\begin{array}{c}\text { Visit } 3 \\
n=9\end{array}$} & \multirow[t]{2}{*}{ Cohen's d } & \multirow[t]{2}{*}{$\begin{array}{c}\text { Friedman } \\
\text { p-value }\end{array}$} \\
\hline & Mean & $\mathbf{S D}^{\star * *}$ & Mean & SD & Mean & SD & & \\
\hline Persons with motor neuron disease & $31.40^{\mathrm{a}}$ & 12.04 & $24.5^{a, b}$ & 10.25 & $21.00^{b}$ & 6.84 & $0.5821^{*}$ & $0.0458^{\star}$ \\
\hline Spouses & $28.53^{a}$ & 11.54 & $20.8^{a, b}$ & 9.74 & $18.77^{b}$ & 9.30 & $0.6387^{\star \star}$ & $0.0151^{*}$ \\
\hline Difference & \multicolumn{2}{|c|}{0.6835} & \multicolumn{2}{|c|}{0.2812} & \multicolumn{2}{|c|}{0.1719} & & 0.8948 \\
\hline Wilcoxon p-value & \multicolumn{2}{|c|}{0.2578} & \multicolumn{2}{|c|}{0.0980} & \multicolumn{2}{|c|}{0.5078} & & \\
\hline
\end{tabular}

Means with different superscripts differ significantly at the $5 \%$ level.

${ }^{*}$ Significance at the $5 \%$ level ${ }^{\star \star}$ Between visit 1 and visit 3 : medium-effect size ${ }^{* \star}$ standard deviation

summary of the obtained results per participant group at each visit, a comparison over time for each group, as well as the difference between the two groups, is presented in Table II.

\section{Communication effectiveness as rated by persons with MND}

A Friedman test was employed to determine whether the change in communication effectiveness, as rated by persons with MND, was statistically significant over time. A p-value of 0.0458 was noted, implying a significant decrease in communication effectiveness between visit 1 and visit 3. The effect size as measured with Cohen's $d$ was medium $(d=0.5821){ }^{23}$

\section{Communication effectiveness as rated by spouses}

In order to determine whether the change in the communication effectiveness of persons with MND as rated by spouses was statistically significant across the visits, a Friedman test was employed. A p-value of 0.0151 was noted, implying that, similar to the persons with MND rating, a statistically significant decrease in communication effectiveness was found between visit 1 and visit 3 . Cohen's $d$ indicated that the size of the effect was medium $(d=$ $0.6387){ }^{23}$

The difference in CETI-M ${ }^{5}$ ratings between persons with MND and spouses at each of the three visits was determined using a Wilcoxon test. As evident from the p-values ( 0.2578 , 0.0980 and 0.5078 , respectively), no statistical significance at the $5 \%$ level was identified (Table II). A comparison of CETI-M ${ }^{5}$ ratings of persons with MND and spouses over time was determined by employing a Friedman test. A $p$-value of 0.8948 was noted, indicating no difference in ratings between the two participant groups over time.

\section{Marital communication}

The $\mathrm{PCl}^{24}$ was administered at each visit to both participant groups. The $\mathrm{PCl}^{24}$ scores have a possible range of 25 to125. Higher scores are indicative of better or more positively viewed marital communication. The mean scores are 105 for "happily" married couples, and 81 for "unhappily" married couples. ${ }^{24}$

Overall, the mean scores of marital communication as perceived by the persons with MND group were consistently higher than those of the spouse group at all three visits. It is important to note that the mean $\mathrm{PCl}^{24}$ scores obtained at the first visit for both these groups were below 105 (the mean score for "happily" married couples). The mean scores decreased across the visits. At the first visit, scores were similar, but the decrease became more marked in the spouse participant group at visit 2 and visit 3, indicating a steady decline to a score of "unhappily" married. At each visit, a summary of the results obtained per participant group, a comparison over time for each group, as well as the difference between the two groups, was recorded, and is presented in Table III.

\section{Perception of marital communication as rated by persons with MND}

A Friedman test was employed to determine whether the change in the perception of marital communication as rated by persons with MND was statistically significant over time.

Table III: Marital communication across visits, and between participant groups

\begin{tabular}{|c|c|c|c|c|c|c|c|c|}
\hline & \multicolumn{2}{|c|}{$\begin{array}{l}\text { Visit } 1 \\
n=14\end{array}$} & \multicolumn{2}{|c|}{$\begin{array}{l}\text { Visit } 2 \\
n=14\end{array}$} & \multicolumn{2}{|c|}{$\begin{array}{c}\text { Visit } 3 \\
n=9\end{array}$} & \multirow{2}{*}{$\begin{array}{l}\text { Cohen's } \\
\quad d\end{array}$} & \multirow{2}{*}{$\begin{array}{c}\text { Friedman } \\
\text { p-value }\end{array}$} \\
\hline & Mean & $\mathbf{S D}^{* * *}$ & Mean & SD & Mean & SD & & \\
\hline Persons with motor neuron disease & 87.99 & 14.15 & 86.00 & 12.54 & 85.22 & 18.49 & - & 0.8233 \\
\hline Spouse & 87.73 & 16.02 & 83.93 & 16.91 & 79.44 & 16.74 & $0.5562^{\star \star}$ & $0.0446^{\star}$ \\
\hline Difference & \multicolumn{2}{|c|}{0.9134} & \multicolumn{2}{|c|}{0.4966} & \multicolumn{2}{|c|}{0.5078} & & 0.2359 \\
\hline Wilcoxon p-value & \multicolumn{2}{|c|}{0.7763} & \multicolumn{2}{|c|}{0.6374} & \multicolumn{2}{|c|}{0.0576} & & \\
\hline
\end{tabular}

\footnotetext{
${ }^{*}$ Significance at the $5 \%$ level ${ }^{* \star}$ Between visit 1 and visit 3: medium-effect size ${ }^{\star \star \star}$ standard deviation
} 
A p-value of 0.8233 was noted, implying that the change was not statistically significant at the $5 \%$ confidence level.

\section{Perception of marital communication as rated by spouses}

The converse was found when examining the change in marital communication as rated by the spouses. A p-value of 0.0446 was noted, implying that there was a statistically significant decrease in the perception of marital communication as indicated by the spouse at the $5 \%$ confidence level between visit 1 and visit 3 . Cohen's $d$ confirmed a medium-effect size $(d=0.5562)$ between visit 1 and visit 3 . There was no statistically significant difference between the marital communication scores of persons with MND and spouses between the visits and across the disease progression.

\section{Speech intelligibility and marital communication}

The primary research aim, namely, to compare how persons with MND and their spouses perceived changes in their marital communication in relation to the deteriorating speech of persons with MND was addressed by employing Spearman's correlation. No significant correlation was found between speech intelligibility and marital communication in either of the participant groups. The most significant finding of the study, that the relationship between the deteriorating speech of persons with MND and the couples' perception of marital communication was not statistically significant across the visits, was unexpected.

\section{Discussion}

It is accepted that when speech intelligibility becomes compromised persons with MND must expend much greater effort to communicate and thus verbal communication is often kept to the minimum to conserve energy. ${ }^{20}$ It was evident that notwithstanding the decline in verbal communication, couples who participated in this study still communicated with each other. As the disease progressed, the majority of persons with MND made use of unaided low-technology AAC to facilitate communication with their spouses. Spouses accepted an increasing share of the communication burden in the conversations in order to facilitate meaning. It is postulated that past shared experiences and familiarity with each other as a couple helped to facilitate each spouse's ability to better interpret facial expressions, gestures and vocalisations and the needs of his or her ill partner. ${ }^{16}$ The ability of spouses to understand the communicative intent and meaning was perceived by the persons with MND as important in the maintenance of social closeness. Reports in the literature allude to the importance of both verbal and nonverbal communication in the maintenance of marital communication, ${ }^{16,17,24}$ but the value of nonverbal communication in maintaining social closeness in the face of decreased verbal communication cannot be underestimated and is supported by the findings of previous studies. ${ }^{20,25}$

The inevitable progressive decline in the speech abilities of persons with MND, despite the type of onset, confirms reports in the literature. As expected, a positive relationship was found across the disease progression between the speech intelligibility and communication effectiveness of persons with MND as rated by both participant groups. The perception of marital communication as indicated by the couples across the disease progression produced unexpected results as no statistically significant difference was reported by the persons with MND, although a statistically significant difference was reported by the spouses across the visits.

Overall, the results prove that the supposition that communication between couples will invariably deteriorate as a result of progressively reduced speech intelligibility is not necessarily valid. The impact of increasingly compromised speech intelligibility which predictably limits verbal communication can be negated by the capacity of the couples to draw on familiarity and past experiences to successfully maintain marital communication.

\section{Limitations of the study}

The small sample size was one of the primary limitations of this study. The unpredictable nature of MND and the unique circumstances of the individual couples impinged on the numbers of participants who could be included in the study. However, the use of alternative statistical measures, such as Cohen's $d$, supports the notion that meaningful effect sizes moderate the need for larger sample sizes. The research design also did not make provision for the retrospective measurement of marital communication. Information on the marital relationship prior to the onset of the MND would have contributed to understanding the complexity of the situation and the role that speech intelligibility played in this regard.

\section{Recommendations for future research}

It is suggested that this study should be replicated using a larger sample size, preferably followed longitudinally. As evidence suggests that the quality of the relationship prior to the onset of illness is a critical determinant of the extent to which chronic illness disrupts the marital relationship, it is suggested that prospective studies are conducted to investigate the impact of speech intelligibility on marital communication in MND. Employing a qualitative research design, e.g. a case study method, to investigate these perceptions could also provide invaluable in-depth 
information to aid in understanding this complex situation that is faced by couples.

\section{Clinical implications and guidance}

Even in the face of loss of speech, communication as a means of facilitating and maintaining the marital relationship is particularly pertinent to persons with MND and their spouses. Therefore, it is important that health professionals provide relief for the relentless physical deterioration, and also take a holistic approach to intervention that also addresses the communication needs of both persons with MND and their spouses. As the value of supporting communication for personal and medical care, community involvement, social interaction and maintaining close marital communication cannot be underestimated, the couple should be provided with appropriate information and support by healthcare professionals. ${ }^{19}$

Guidelines for intervention that address the communication needs of persons with MND and their family include: ${ }^{9}$

- Appropriate timing of referral to an AAC specialist or speech therapist for screening, assessment, intervention and education. This is one of the most important clinical decisions that are essential to ensure that when residual speech is no longer effective, AAC strategies are already in place.

- The AAC specialist or speech therapist should clinically monitor the speaking rate using a measure such as the SIT. ${ }^{23}$ Generally, it is accepted that once the speaking rate is less than 125 words per minute, AAC strategies should be implemented. This allows gradual familiarisation with the AAC strategies. ${ }^{9}$

- Speech intervention, that includes oral exercises to improve strength and mobility, should be discouraged.

- Speech intervention should rather focus on providing strategies to persons with MND and the communicative partners that conserve energy for priority speaking tasks, reduce fatigue and effort, e.g. using a voice amplifier when speaking in noisy situations, and avoid communicating in adverse communication situations, such as noisy, crowded rooms.

\section{Declaration}

This research was partially funded by the National Research Fund (NRF) and the Carnegie Corporation of New York. The authors alone are responsible for the content and writing of the paper.

\section{References}

1. Miglioretti M, Mazzini L, Oggioni GD, et al. Illness perceptions, mood and healthrelated quality of life in patients with amytrophic lateral sclerosis. J Psychosom Res. 2008;65(6):603-609.
2. Logroscino G, Traynor BJ, Hardiman O, et al. Descriptive epidemiology of amyotrophic lateral sclerosis: new evidence and unsolved issues. J Neurol Neurosurg Psychiatry. 2008;79(1):6-11.

3. Doyle M, Phillips B. Trends in augmentative and alternative communication use by individuals with amyotrophic lateral sclerosis. Augmen Altern Commun. 2001;17(3):167-178.

4. World Health Organization. International Classification of Functioning, Disability and Health (ICF). Geneva: WHO; 2001.

5. Yorkston KM, Beukelman DR, Strand EA, Bell KR. Management in motor speech disorders in children and adults. $2^{\text {nd }}$ ed. Austin: Pro-Ed; 1999.

6. Light JC, Arnold KB, Clark EA. Finding a place in the 'social circle of life'. In: Light $\mathrm{JC}$, Beukelman DR, Reichle J, editors. Communicative competence for individuals who use AAC: from research to effective practice. Baltimore: Paul H Brookes Publishing, 2003; p. 361-397.

7. Ball LJ, Beukelman DR, Pattee GL. Communication effectiveness of individuals with amyotrophic lateral sclerosis. J Commun Disord. 2004;37(3):197-215.

8. Darley F, Aronson A, Brown J. Clusters of deviant speech dimensions in the dysarthrias. J Speech Lang Hear Disord. 1969;12(3):462-496.

9. Beukelman DM, Fager S, Nordness A. Communication support for people with ALS. Neurol Res International vol 2011, Article ID 714693, 6 pages, 2011. doi:10.1155/2011/714693.

10. Yunusova $\mathrm{Y}$, Weismer G, Kent RD, Rusche NM. Breath-group intelligibiity in dysarthria: characteristics and underlying correlates. J Speech Lang Hear Res. 2005;48(6):1294-1310.

11. Light J. Toward a definition of communicative competence for individuals using augmentative and alternative communication systems. Augmen Altern Commun. 1989;5(2):137-144.

12. Ball LJ, Willis A, Beukelman DR, Pattee G L. A protocol for the identification of early bulbar signs in amyotrophic lateral sclerosis. J Neurol Sci. 2001;191(1-2):43-53.

13. Mathy $P$, Yorkston KM, Gutmann ML. AAC for individuals with amyotrophic lateral sclerosis. In: Beukelman DR, Yorkston KM, Reichle J, editors. Augmentative and alternative communication for adults with acquired neurological disorders. Baltimore: Paul H Brookes Publishing, 2000; p.183-232.

14. Beukelman DR, Ball LJ, Fager S. An AAC personnel framework: adults with acquired complex communication needs. Augmen Altern Commun. 2008;24(3):255-267.

15. Ross E, Deverell A. Psychosocial approaches to health, illness and disability. A reader for health care professionals. Pretoria: Van Schaik Publishers; 2004.

16. Cutrona CE. A psychological perspective: marriage and the social provisions of relationships. J Marriage Fam. 2004;66:992-999.

17. Rolland JS. Cancer and the family: an integrative model. Cancer. 2005;104(Suppl 11):2584-2595.

18. Kahn M. Non-verbal communication and marital satisfaction. Fam Process. 1970;9(4):449-456.

19. Beukelman DR, Yorkston KM. Augmentative and alternative communication application for persons with severe acquired communication disorders: an introduction. Augmen Altern Commun.1998;5(1):42-48.

20. Murphy J. "I prefer contact this close": perceptions of AAC by people with motor neuron disease and their communication partners. Augmen Altern Commun. 2004;20(4):259-271.

21. Riviere $M$, Meininger $V$, Zeisser $P$, Munsat $T$. An analysis of extended survival in patients with amyotrophic lateral sclerosis treated with riluzole. Arch Neurol. 1998;55(4):526-548

22. Hillel AD, Miller RM, Yorkston K, et al. Amyotrophic lateral sclerosis severity scale. Neuroepidemiology. 1989;8(3):142-150.

23. Yorkston KM, Beukelman DR, Tice R. Sentence intelligibility test for Windows. Lincoln: Institute for Rehabilitation Science and Engineering, Madonna Rehabilitation Hospital; 1996.

24. Navran L. Communication and adjustment in marriage. Fam Process. 1967;6:173-184.

25. Fried-Oken M, Fox L, Rau MT, et al. Purposes of AAC device use for PALS as reported by caregivers. Augmen Altern Commun. 2006;22(3):209-221. 